\title{
Liver Contusion
}

National Cancer Institute

\section{Source}

National Cancer Institute. Liver Contusion. NCI Thesaurus. Code C50853.

Bruising of the liver. 